\title{
ON THE RF-PAIR OPERATIONS FOR THE EXACT SOLUTION OF SOME CLASSES OF NONLINEAR VOLTERRA INTEGRAL EQUATIONS
}

\author{
P. DARANIA AND M. HADIZADEH
}

Received 1 May 2005; Revised 17 November 2005; Accepted 15 January 2006

We study the exact solution of some classes of nonlinear integral equations by series of some invertible transformations and $R F$-pair operations. We show that this method applies to several classes of nonlinear Volterra integral equations as well and give some useful invertible transformations for converting these equations into differential equations of Emden-Fowler type. As a consequence, we analyze the effect of the proposed operations on the exact solution of the transformed equation in order to find the exact solution of the original equation. Some applications of the method are also given. This approach is effective to find a great number of new integrable equations, which thus far, could not be integrated using the classical methods.

Copyright (c) 2006 P. Darania and M. Hadizadeh. This is an open access article distributed under the Creative Commons Attribution License, which permits unrestricted use, distribution, and reproduction in any medium, provided the original work is properly cited.

\section{Introduction}

Many problems in mathematical physics, contact problems in the theory of elasticity, and mixed boundary value problems are transformed into nonlinear Volterra integral equations (see $[2,8,12])$. The theory and application of integral equations is an important subject within applied mathematics. Due to the fact that this equation appears in many branches of physics and engineering, for example, in stellar dynamics, quantum mechanics, and fluid dynamics, there is great interest in finding both exact or closed form approximate solutions. (see $[6,7,11]$ ).

Exact solutions have always played an important role in properly understanding the qualitative features of many phenomena and processes in various fields of natural science. On the other hand, equations of applied and theoretical physics often contain parameters or functions which are found experimentally and therefore are not stringently fixed. At the same time, equations that model real phenomena and processes must be sufficiently simple to make possible their analysis and solution. It is natural to adopt, as the criterion of simplicity, the requirement that the model equation admits a solution in

Hindawi Publishing Corporation

Mathematical Problems in Engineering

Volume 2006, Article ID 97020, Pages 1-11

DOI 10.1155/MPE/2006/97020 
a closed form. It should be noted that even particular exact solutions of nonlinear equation (including those without a clear physical sense and which do not correspond to real phenomena and processes) play an important role of test problems for verifying the correctness and assessment of accuracy of various numerical, asymptotic, and approximate methods. Moreover, the model equations and problems admitting exact solutions serve as the basis for the development of new numerical, asymptotic, and approximate methods which in turn, enable us to investigate more complicated problems having no analytical solution.

There are many problems in astrophysics, gas and fluid dynamics, nuclear physics, and chemical reactions in which Emden-Fowler equation arises. A summary of the historical developments of the Emden-Fowler equation may be found in [4, 10, 16-18] where an excellent bibliography on its applications in mechanics and physics is also given in [21].

Note that, closed form solutions of Emden-Fowler equations are very scarce and the search for the conservation which can in many respects shed light on the physical mechanism of the dynamical problem has its full vindication. However, all the authors have applied various methods which are generally based upon the group theoretical method, the method of integrating factors, Noetherian approach method, and so forth. See $[1,3$, $5,9,13,19]$.

In this paper, by means of appropriate transformations of $R F$-pair operations, some classes of nonlinear integral equations are transformed into some solvable differential equations of Emden-Fowler class. For this purpose, we give some useful invertible relations between the Emden-Fowler and the special class of nonlinear Volterra integral equations.

The outline of this paper is as follows. In Section 2, we will give some basic definitions and elementary results which have been so far obtained. In Section 3, we will be mainly concerned with the nonlinear Volterra integral equations, in order to convert them to the classical Emden-Fowler equations. Finally, in Section 4 some applications of the method are presented which are particularly relevant in mathematical physics. This approach is effective to find a great number of new integrable equations which thus far, could not be integrated using the classical methods.

\section{Preliminaries}

Here, we will state some elementary concepts and definitions, which our results are based on.

Definition 2.1. The class of generalized Emden-Fowler equations is written as

$$
y_{x x}^{\prime \prime}=A x^{n} y^{m}\left(y_{x}^{\prime}\right)^{l}, \quad v=(n, m, l),
$$

where it is determined by a three-dimensional parameter vector $v=(n, m, l) \in R^{3}$, and the parameter $A$ is insignificant. The parameter subspace $(n, m, 0)$ defines the set of classical Emden-Fowler equations,

$$
y_{x x}^{\prime \prime}=A x^{n} y^{m}
$$


Definition 2.2. An $R F$-pair is an operation of consecutive raising and lowering of the order of equation. (The term $R F$-pair is formed by the initial letters of the words "Rise" and "Fall".)

Now, we define the following $R$-operations and $F$-operations:

(i) termwise $m$-fold differentiation of the original equation, type $R D^{m}$,

(ii) termwise one-or two-fold differentiation of original equation with respect to the independent variable, type accordingly $R X$ or $R X^{2}$,

(iii) the equation is an exact derivative of the $m$ th-order termwise integration $m$ times, type $F I^{m}$,

(iv) the equation is autonomous, that is, it does not conclude an independent variable in an explicit form, type $F X$ :

$$
F X: y_{x}^{\prime}=u(y), \quad y_{x x}^{\prime \prime}=u u_{y}^{\prime}
$$

(v) the equation is homogeneous in the extended sense, type $F U$ : the transformation $x=e^{t}, y=u e^{k t}$, with an appropriate choice of $k$, leads to an autonomous form followed by a transformation $F X$.

Note that if an $R(F)$-operating $R Z^{m}\left(F Z^{m}\right)$ is inverted, it is denoted by $R Z^{-m}\left(F Z^{-m}\right)$.

\section{Some relations between the Emden-Fowler equations and nonlinear Volterra integral equations}

In order to show how the original equation is transformed into a differential equation of Emden-Fowler type, we first consider the following nonlinear Volterra integral equation:

$$
y(x)=f(x)+\lambda \int_{0}^{x} g(y(s)) d s .
$$

Actually few theoretical methods in some several cases for this equation are known. Detailed descriptions and analysis of these methods may be found in [14] and references therein.

Here, we will consider the special case of (3.1), when the form of nonlinearity is $g(y(s))=y^{1 / 2}(s)$ and we will give some useful invertible relations between the EmdenFowler and the nonlinear Volterra integral equation (3.1).

The main purpose of this section is to prove the following theorems by using the $R F$ pair operations.

Theorem 3.1. Application of the RF-pair operations to the classical Emden-Fowler equation,

$$
y_{x x}^{\prime \prime}=A x^{n} y^{m}, \quad v=(n, m, 0),
$$

where $m, n$ are arbitrary real numbers and $m \neq \pm 1$, gives rise to the nonlinear Volterra integral equation

$$
z(u)=\frac{2 A}{m+1} u^{m+1}-k(k-1) u^{2}+c+2(1-2 k) \int_{0}^{u} z^{1 / 2}(s) d s,
$$


4 On the $R F$-pair operations for integral equations

where $c$ is a constant and $k$ is an arbitrary real number which can be suitably chosen in order to convert the original equation into an autonomous equation. Conversely (3.3) implies (3.2).

Proof. Applying the operation $F U: x=e^{t}, y=u e^{k t}$ into (3.2) leads to the equation

$$
u_{t t}^{\prime \prime}+(2 k-1) u_{t}^{\prime}+k(k-1) u=A u^{m} e^{t(k(m-1)+n+2)} .
$$

This equation, by choosing $k=-(n+2) /(m-1)$, reduces to the autonomous equation

$$
u_{t t}^{\prime \prime}+(2 k-1) u_{t}^{\prime}+k(k-1) u=A u^{m}
$$

and by using the operation $F X: u_{t}^{\prime}=q(u), u_{t t}^{\prime \prime}=q q_{u}^{\prime}$, (3.5) reduces to the following equation:

$$
q q_{u}^{\prime}+(2 k-1) q+k(k-1) u=A u^{m}
$$

Now, by applying the operator FI into (3.6), we obtain

$$
q^{2}(u)=\frac{2 A}{m+1} u^{m+1}-k(k-1) u^{2}+q^{2}(0)-2(2 k-1) \int_{0}^{u} q(s) d s
$$

in which the substitution $q^{2}(u)=z(u), q^{2}(0)=c$, gives the following nonlinear Volterra integral equation:

$$
z(u)=f(u)+\lambda \int_{0}^{u} z^{1 / 2}(s) d s
$$

where

$$
f(u)=\frac{2 A}{m+1} u^{m+1}-k(k-1) u^{2}+c, \quad \lambda=2(1-2 k) .
$$

Conversely, it is evident that (3.3), by substituting $z(u)=q^{2}(u)$ and successive use of operators $F I^{-1}, X^{-1}, F U^{-1}$ reduces to the original equation (3.2).

Remark 3.2. Here, and in the rest of this paper, we note that the exact solutions of the reference equation and transformed equation correspond to each other. Hence, if the exact solution of the transformed equation is unique, so is the exact solution of the reference equation.

Remark 3.3. Note that, in Theorem 3.1, we considered the case $m \neq \pm 1$. Now for the cases $m=1,-1$, we have the following theorems, respectively.

Theorem 3.4. The classical Emden-Fowler equation,

$$
y_{x x}^{\prime \prime}=A x^{n} y, \quad v=(n, 1,0)
$$


is transformed into the Volterra integral equation

$$
z(u)=(A-k(k-1)) u^{2}+c-2(2 k-1) \int_{b}^{u} z^{1 / 2}(s) d s,
$$

if and only if $n=-2$.

Proof. By using the operator FU, (3.10) is reduced to the following equation:

$$
u_{t t}^{\prime \prime}+(2 k-1) u_{t}^{\prime}+k(k-1) u=A u e^{t(n+2)},
$$

and this equation is an autonomous equation if and only if $n=-2$. So, it is transformed into the following autonomous equation:

$$
u_{t t}^{\prime \prime}+(2 k-1) u_{t}^{\prime}+k(k-1) u=A u
$$

where $k$ is an arbitrary constant. Applying operator $F X$ on (3.13) gives

$$
q q_{u}^{\prime}+(2 k-1) q=(A-k(k-1)) u
$$

Now, by using the operator FI (integrated from $b$ to $u$, where $u$ is a variable and $b$ is a constant) on (3.14), and substituting $q^{2}(u)=z(u)$ and $q^{2}(b)=c$ we get

$$
z(u)=(A-k(k-1)) u^{2}+c-2(2 k-1) \int_{b}^{u} z^{1 / 2}(s) d s .
$$

Conversely, it is evident that (3.15), by substituting $z(u)=q^{2}(u)$ and using the operators $F I^{-1}, F X^{-1}$, and $F U^{-1}$ successively, is reduced to the original equation (3.10).

Theorem 3.5. The classical Emden-Fowler equation,

$$
y_{x x}^{\prime \prime}=A x^{n} y^{m}, \quad v=(n,-1,0)
$$

is transformed to the Volterra integral equation

$$
z(u)=k(1-k) u^{2}+A \ln u+c-2(2 k-1) \int_{b}^{u} z^{1 / 2}(s) d s
$$

and conversely.

Proof. We refrain from going into details and using a similar procedure as outlined in the proof of Theorem 3.4, the equation $y_{x x}^{\prime \prime}=A x^{n} y^{m}$ in this case is equivalent to the equation $y^{\prime \prime}=A x^{n} y^{-1}$. This equation, by applying the successive use of operators $F U, F X$, and $F I$, reduces to $(3.17)$ where $k=(n+2) /(2)$.

Note that, all the $R F$-pair operations and transformations are invertible and under the $R F$-pair operations, the solution of the transformed equation can be converted into the 
6 On the $R F$-pair operations for integral equations

solution of the reference equation. So, due to solvability of some classes of Emden-Fowler equations $[15,22]$, the proposed integral equation is solvable in the case considered.

3.1. Generalization to integral equations of convolution type. The arguments of Section 3 can readily be extended to other types of Volterra integral equations, for example, the nonlinear Volterra integral equations with convolution kernels:

$$
z(u)=f(u)+\lambda \int_{0}^{u}(u-t) z^{1 / 2}(t) d t
$$

In relation of this type of equations we have the following result.

THEOREM 3.6. By application of the RF-pair operations, the convolution integral equation (3.18) with $f(u)=a u+b$, where $a, b$ are constants, reduces to the solvable class of classical Emden-Fowler equation,

$$
p_{t t}^{\prime \prime}=\frac{\lambda}{2} p^{2}, \quad v=(0,2,0)
$$

and conversely.

Proof. Consider the integral equation (3.18) and let $z(u)=q^{2}(u)$, then by applying the operator $R X^{2}$, we obtain

$$
q_{u}^{\prime 2}+q q_{u u}^{\prime \prime}=\frac{1}{2} f_{u u}^{\prime \prime}+\frac{\lambda}{2} q(u)
$$

Using the operator $F X^{-1}$ on $(3.20)$, we have

$$
\frac{u_{t t t}^{\prime \prime \prime}}{u_{t}^{\prime}}=\frac{1}{2} f_{u u}^{\prime \prime}+\frac{\lambda}{2} u_{t}^{\prime} .
$$

Then by the effect of the operator $R P: u_{t}^{\prime}=p(t), u_{t t}^{\prime \prime}=p_{t}^{\prime}, u_{t t t}^{\prime \prime \prime}=p_{t t}^{\prime \prime}$, upon (3.21), we get

$$
p_{t t}^{\prime \prime}=\frac{1}{2} p f_{u u}^{\prime \prime}+\frac{\lambda}{2} p^{2}
$$

The assumption $f(u)=a u+b$ implies that $f_{u u}^{\prime \prime}=0$ and hence (3.22) is equivalent to the classical Emden-Fowler equation (3.19) which is solvable according to [15, 19]. (For further details see $[15,19,22]$.)

Conversely, appealing to the properties of the $R F$-pair operation, we can get the integral equation (3.18) by applying the operators $R P^{-1}, F X^{-1}, F I^{2}$ successively on (3.19).

\section{Some applications and further comments}

As applications of the proposed scheme, we give in this section some examples and applications of the procedure, which is of particular interest in mathematical physics [3], control process [20], and nonlinear mechanics [21]. 
(1) Let us consider the Emden-Fowler equation (3.2), where $n=-m-3$ and $m \neq \pm 1$, $m \in R$ which is of special interest in [20, page 255]. In view of Theorem 3.1, (3.2) is reduced to the solvable equation

$$
y_{x x}^{\prime \prime}=A x^{-m-3} y^{m}, \quad v=(-m-3, m, 0),
$$

with parametric exact solution

$$
x=a C_{1}^{h} \varphi\left(\tau, C_{2}\right)=a C_{1}^{h} E_{m}^{-1}, \quad y=b C_{1}^{l} \psi\left(\tau, C_{2}\right)=b C_{1}^{l} \tau E_{m}^{-1},
$$

where

$$
\begin{gathered}
A= \pm \frac{m+1}{2} a^{m+1} b^{1-m}, \quad h=m-1, \quad l=m+1, \\
E_{m}=\int\left(1 \pm \tau^{m+1}\right)^{-1 / 2} d \tau+C_{2}, \quad \psi=\tau E_{m}^{-1}, \quad \varphi=E_{m}^{-1},
\end{gathered}
$$

and $C_{1}, C_{2}, a, b$ are constants, $A=f(a, b)$ is an insignificant parameter: this is fully discussed in [21, page 254]. Hence by this assumption, the integral equation (3.3) is reduced to

$$
z(u)=\frac{2 A}{m+1} u^{m+1}-\frac{2(m+1)}{(m-1)^{2}} u^{2}+c-2\left(\frac{m+3}{m-1}\right) \int_{0}^{u} z^{1 / 2}(s) d s .
$$

Now, by applying the operation $F U: x=e^{t}, y=u e^{k t}$ to the relations (4.2), we obtain that

$$
\begin{gathered}
t=\ln \left(a C_{1}^{h} E_{m}^{-1}\right), \\
u=a^{-k} b C_{1}^{l-k h} \tau E_{m}^{k-1},
\end{gathered}
$$

where $k=(m+1) /(m-1)$.

The relations (4.6) and (4.7) yield

$$
\begin{gathered}
t_{\tau}^{\prime}=-E_{m}^{-1} \frac{d}{d \tau} E_{m} \\
u_{\tau}^{\prime}=a^{-k} b C_{1}^{l-k h} E_{m}^{k-1}\left(1+(k-1) \tau E_{m}^{-1} \frac{d}{d \tau} E_{m}\right) .
\end{gathered}
$$

Also, we have

$$
u_{t}^{\prime}=\frac{u_{\tau}^{\prime}}{t_{\tau}^{\prime}}=a^{-k} b C_{1}^{l-k h} E_{m}^{k-1}\left(1+(k-1) \tau E_{m}^{-1} \frac{d}{d \tau} E_{m}\right) .
$$

Then using the operation $F X: u_{t}^{\prime}=q(u)$ and relations (4.6) and (4.7), we obtain

$$
q(u)=-\frac{a^{-k} b C_{1}^{l-k h} E_{m}^{k}\left(1+(k-1) \tau E_{m}^{-1}(d / d \tau) E_{m}\right)}{(d / d \tau) E_{m}}
$$


8 On the $R F$-pair operations for integral equations

where $u$ is defined as (4.7) and

$$
\frac{d}{d \tau} E_{m}=\left(1 \pm \tau^{m+1}\right)^{-1 / 2}
$$

Now, putting $z(u)=q^{2}(u)$, we get the exact solution for the integral equation (4.5) in the following form:

$$
z(u)=a^{-2 k} b^{2} C_{1}^{2(l-k h)}\left(1 \pm \tau^{m+1}\right) E_{m}^{2 k}\left(1+(k-1) \tau E_{m}^{-1} \frac{d}{d \tau} E_{m}\right)^{2},
$$

where $u$ is defined as (4.7).

Remark 4.1. We can compute $E_{m}$ in (4.12), analytically, in the following manner: if $m=$ $-(2 j-1) /(2 j+1)($ or $(1-j) / j)$, where $j \in \mathscr{L}-\{0\}$, then using the transformation $1 \pm$ $\tau^{m+1}=t^{2} \tau^{m+1}$ (or $\left.t^{2}\right), E_{m}$ is integrable by the classical methods. For example, if we set $j=1$, then $m=-1 / 3$, and hence (4.5) is transformed to the following integral equation:

$$
z(u)=3 A u^{2 / 3}-\frac{3}{4} u^{2}+c+4 \int_{0}^{u} z^{1 / 2}(s) d s .
$$

Now, for obtaining the analytical solution of the above equation, according to (4.3), we consider two cases for parameter $A$.

Case 1. If $A=(1 / 3) a^{2 / 3} b^{4 / 3}$, then (4.13) is reduced to

$$
z(u)=a^{2 / 3} b^{4 / 3} u^{2 / 3}-\frac{3}{4} u^{2}+c+4 \int_{0}^{u} z^{1 / 2}(s) d s .
$$

Also, we need to compute

$$
E_{-1 / 3}=\int\left(1-\tau^{2 / 3}\right)^{-1 / 2} d \tau
$$

and to do this, by substituting the transformation $1-\tau^{2 / 3}=t^{2} \tau^{2 / 3}$ such that

$$
d \tau=-3 t\left(t^{2}-1\right)^{-5 / 2} d t
$$

into $E_{-1 / 3}$, we have

$$
E_{-1 / 3}=-3 \int \frac{d t}{\left(t^{2}-1\right)^{2}}+C_{2}=\frac{3}{2} \tau^{2 / 3}\left(\tau^{-2 / 3}+1\right)^{1 / 2}+\frac{3}{4} \ln \left(\frac{\left(\tau^{-2 / 3}+1\right)^{1 / 2}-1}{\left(\tau^{-2 / 3}+1\right)^{1 / 2}+1}\right)+C_{2} .
$$

Substituting $E_{-1 / 3}$ in (4.12), we obtain the parametric exact solution of (4.14) in the form

$$
z(u)=a^{1 / 2} b \tau\left[\frac{3}{2} \tau^{2 / 3}\left(\tau^{2 / 3}+1\right)^{1 / 2}+\frac{3}{4} \ln \left(\frac{\left(\tau^{-2 / 3}+1\right)^{1 / 2}-1}{\left(\tau^{-2 / 3}+1\right)^{1 / 2}+1}\right)+C_{2}\right]^{-3 / 2},
$$


where

$$
u=a^{1 / 2} b \tau\left(E_{-1 / 3}\right)^{-3 / 2}
$$

and $a, b$ are defined by an insignificant parameter $A$.

Case 2. If $A=(-1 / 3) a^{2 / 3} b^{4 / 3}$, using a similar procedure as outlined in Case 1 , we have

$$
\begin{gathered}
z(u)=-a^{2 / 3} b^{4 / 3} u^{2 / 3}-\frac{3}{4} u^{2}+c+4 \int_{0}^{u} z^{1 / 2}(s) d s \\
E_{-1 / 3}=-3 \int \frac{d t}{\left(t^{2}+1\right)^{2}}+C_{2}=-\frac{3}{2} \tau^{2 / 3}\left(\tau^{-2 / 3}-1\right)^{1 / 2}-\frac{3}{2} \arctan \left(\tau^{-2 / 3}-1\right)^{1 / 2}+C_{2} .
\end{gathered}
$$

Here, by substituting $E_{-1 / 3}$ in (4.12), we get the exact parametric solution of integral equation (4.20) in the form

$$
z(u)=a^{1 / 2} b \tau\left[-\frac{3}{2} \tau^{2 / 3}\left(\tau^{2 / 3}-1\right)^{1 / 2}-\frac{3}{2} \arctan \left(\tau^{-2 / 3}-1\right)^{1 / 2}\right]^{-3 / 2}
$$

where $u$ and $a, b$ are defined as (4.19) and an insignificiant parameter $A$, respectively.

(2) In order to exhibit other applications of Theorem 3.1, we consider the nonlinear Volterra integral equation

$$
z(u)=-6 u^{2}-2 u+\frac{1}{9}-10 \int_{0}^{u} z^{1 / 2}(s) d s,
$$

and appealing to Theorem 3.1, we have $m=0, n=1, k=3, c=1 / 9$, and $A=-1$, as a consequence of which this equation is reduced to the solvable Emden-Fowler equation

$$
u_{x x}^{\prime \prime}=-x
$$

where one of the exact solutions of this equation is

$$
u(x)=x-\frac{1}{6} x^{3}
$$

Now by effecting the operations $F U$ and FX successively to the solution (4.25), we obtain the exact solution of the reference integral equation with respect to the exact solution of the transformed equation in the form

$$
z(u)=4 u^{2}+\frac{4}{3} u+\frac{1}{9}
$$

(3) In view of Theorem 3.4, (3.10) is equivalent to the solvable class of Emden-Fowler equation,

$$
y^{\prime \prime}=A x^{-2} y
$$


with parametric exact solution,

$$
x=\tau^{2}, \quad y=\tau\left(c_{1} \tau^{\nu}+c_{2} \tau^{-v}\right),
$$

where $c_{1}$ and $c_{2}$ are constants and $\nu=\sqrt{1+4 A}, 1+4 A>0$ (see [21, page 263] and [15]). In this case, the integral equation (3.15) is equivalent to the equation

$$
z(u)=(A-k(k-1)) u^{2}+c-2(2 k-1) \int_{0}^{u} z(s)^{1 / 2} d s .
$$

Now, for computing the exact solution of this equation, by applying the operation $F U$ on the relations (4.28), we get

$$
\begin{gathered}
e^{t}=\tau^{2}, \\
u=\tau^{1-2 k}\left(c_{1} \tau^{v}+c_{2} \tau^{-v}\right),
\end{gathered}
$$

and using the operation $F X$ on (4.30), it follows that

$$
q(u)=\frac{1}{2}(1-2 k) \tau^{1-2 k}\left(c_{1} \tau^{\nu}+c_{2} \tau^{-\nu}\right)+\frac{1}{2} \tau^{2-2 k}\left(c_{1} \nu \tau^{\nu-1}-c_{2} \nu \tau^{-\nu-1}\right) .
$$

In this position, by substitution $z(u)=q^{2}(u)$ in (4.31), we can get the parametric exact solution of the integral equation (4.29) in the following form:

$$
z(u)=\left[\frac{1}{2}(1-2 k) \tau^{1-2 k}\left(c_{1} \tau^{\nu}+c_{2} \tau^{-\nu}\right)+\frac{1}{2} \tau^{2-2 k}\left(c_{1} \nu \tau^{\nu-1}-c_{2} \nu \tau^{-\nu-1}\right)\right]^{2},
$$

where $u$ is defined as (4.30).

\section{Conclusion}

This approach can reveal that, under the $R F$-pair operations, the solution of the differential equation of the Emden-Fowler type can be transformed into the solution of the nonlinear Volterra integral equation. The action of the operations upon an equation of the class which has been investigated, reduces the equation in some other standard class which may be integrated using classical methods.

\section{Acknowledgments}

The authors would like to thank the anonymous referees for carefully reading and commenting on this paper. This research was in part supported by a Grant from IPM (no. 83650039).

\section{References}

[1] M. B. Abd-el-Malek, Application of the group-theoretical method to physical problems, Journal of Nonlinear Mathematical Physics 5 (1998), no. 3, 314-330.

[2] M. A. Abdou, On the solution of linear and nonlinear integral equation, Applied Mathematics and Computation 146 (2003), no. 2-3, 857-871. 
[3] L. M. Berkovich, The generalized Emden-Fowler equation, Symmetry in Nonlinear Mathematical Physics 1 (1997), 155-163.

[4] S. Chandrasekhar, An Introduction to the Study of Stellar Structure, Dover, New York, 1967.

[5] H. S. M. Coxeter and W. O. J. Moser, Generators and Relations for Discrete Groups, Springer, New York, 1972.

[6] H. T. Davis, Introduction to Nonlinear Differential and Integral Equations, Dover, New York, 1962.

[7] L. M. Delves and J. L. Mohamed, Computational Methods for Integral Equations, Cambridge University Press, Cambridge, 1985.

[8] M. Ganesh and M. C. Joshi, Numerical solvability of Hammerstein integral equations of mixed type, IMA Journal of Numerical Analysis 11 (1991), no. 1, 21-31.

[9] P. M. Lima, Numerical methods and asymptotic error expansions for the Emden-Fowler equations, Journal of Computational and Applied Mathematics 70 (1996), no. 2, 245-266.

[10] C. D. Luning and W. L. Perry, Positive solutions of negative exponent generalized Emden-Fowler boundary value problems, SIAM Journal on Mathematical Analysis 12 (1981), no. 6, 874-879.

[11] N. M. Madbouly, D. F. McGhee, and G. F. Roach, Adomian's method for Hammerstein integral equations arising from chemical reactor theory, Applied Mathematics and Computation 117 (2001), no. 2-3, 241-249.

[12] R. K. Miller, Nonlinear Volterra Integral Equations, Mathematics Lecture Note Series, W. A. Benjamin, California, 1971.

[13] L. V. Ovsiannikov, Group Analysis of Differential Equations, Academic Press, New York, 1982.

[14] A. D. Polyanin and A. V. Manzhirov, Handbook of Integral Equations, CRC Press, Florida, 1998.

[15] A. D. Polyanin and V. F. Zaitsev, Handbook of Exact Solutions for Ordinary Differential Equations, 2nd ed., Chapman \& Hall/CRC, Florida, 2003.

[16] B. D. Vujanovic, A. M. Strauss, S. E. Jones, and P. P. Gillis, Polynomial conservation laws of the generalized Emden-Fowler equation, International Journal of Non-Linear Mechanics 33 (1998), no. 2, 377-384.

[17] A.-M. Wazwaz, A new algorithm for solving differential equations of Lane-Emden type, Applied Mathematics and Computation 118 (2001), no. 2-3, 287-310.

[18] J. S. W. Wong, On the generalized Emden-Fowler equation, SIAM Review 17 (1975), no. 2, 339360.

[19] V. F. Zaitsev, On discrete group analysis of ordinary differential equations, Soviet Mathematics. Doklady 37 (1988), no. 2, 403-406.

[20] V. F. Zaitsev and A. V. Flegontov, Creation of new methods of mathematical physics, search of the exact solutions and first integrals of nonlinear differential equations, Differential Equations and Control Process (1997), no. 1, 252-260.

[21] V. F. Zaitsev and A. D. Polyanin, Discrete Group Methods for Integrating Equations of Nonlinear Mechanics, CRC Press, Florida, 1994.

[22] http://eqworld.ipmnet.ru/ (The World of Mathematical Equations).

P. Darania: Department of Mathematics, Urmia University, P.O. Box 165, Urmia, Iran

E-mail address: p.darania@mail.urmia.ac.ir

M. Hadizadeh: Department of Mathematics, K. N. Toosi University of Technology, Tehran 16315, Iran; Institute for Studies in Theoretical Physics and Mathematics (IPM), P.O. Box 19395-5746,

Tehran, Iran

E-mail address: hadizadeh@kntu.ac.ir 


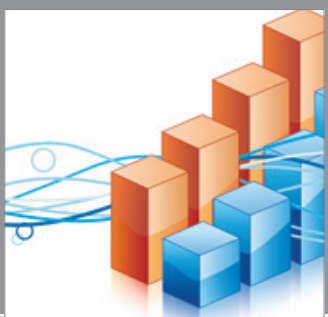

Advances in

Operations Research

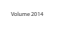

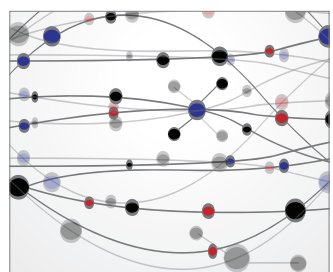

\section{The Scientific} World Journal
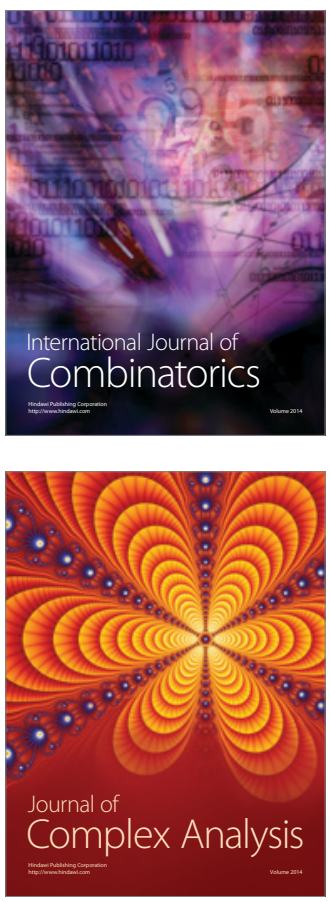

International Journal of

Mathematics and

Mathematical

Sciences
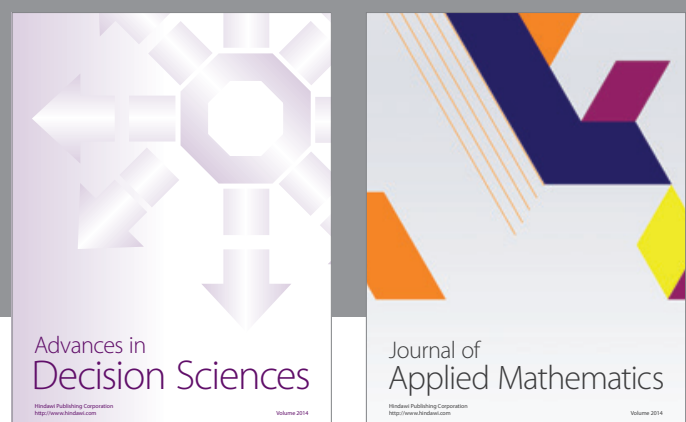

Journal of

Applied Mathematics
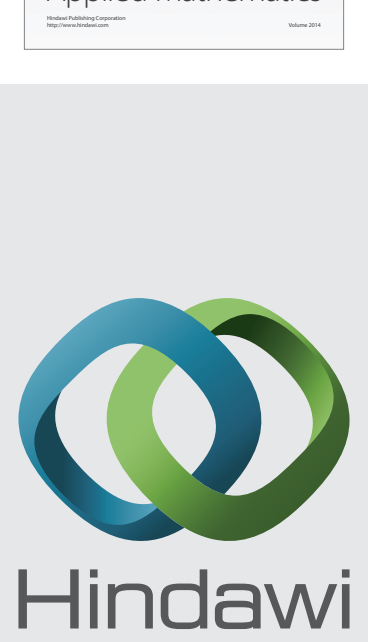

Submit your manuscripts at http://www.hindawi.com
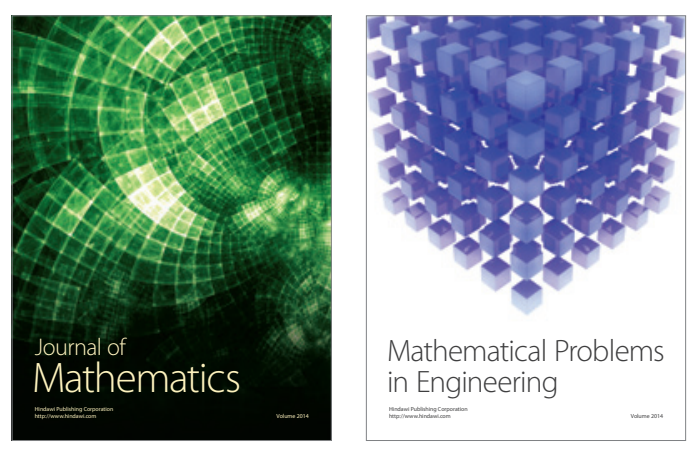

Mathematical Problems in Engineering
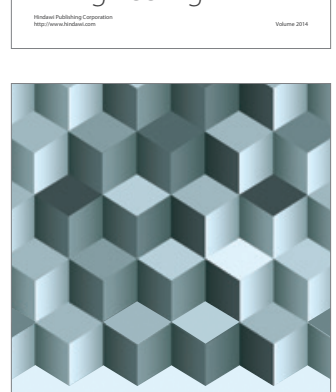

Journal of

Function Spaces
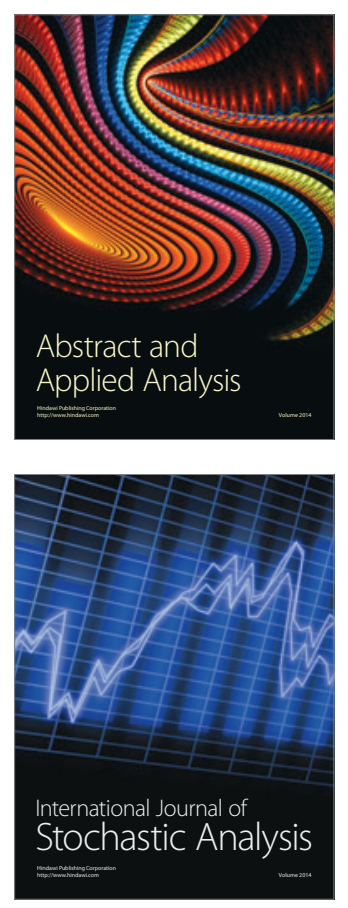

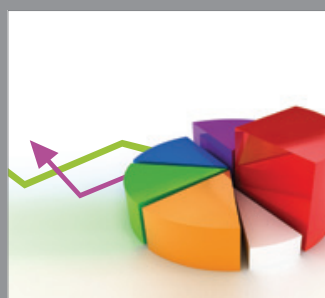

ournal of

Probability and Statistics

Promensencen
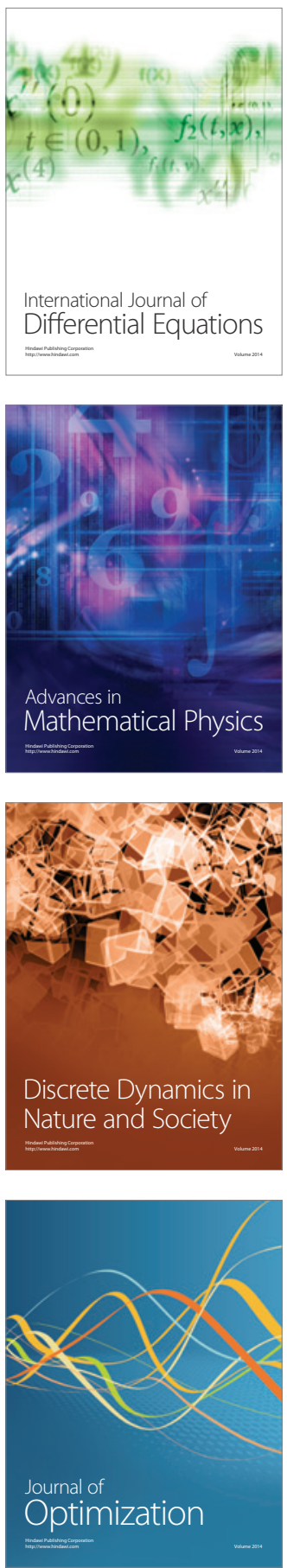